\title{
GCU
}

Glasgow Caledonian

University

University for the Common Good

\section{Application of K-Means method to pattern recognition in on-line cable partial discharge monitoring}

Peng, Xiaosheng; Zhou, Chengke; Hepburn, Donald; Judd, Martin; Siew, W H

Published in:

IEEE Transactions on Dielectrics and Electrical Insulation

DOI:

10.1109/TDEI.2013.6518945

Publication date:

2013

Document Version

Author accepted manuscript

Link to publication in ResearchOnline

Citation for published version (Harvard):

Peng, X, Zhou, C, Hepburn, D, Judd, M \& Siew, WH 2013, 'Application of K-Means method to pattern recognition in on-line cable partial discharge monitoring', IEEE Transactions on Dielectrics and Electrical Insulation, vol. 20, no. 3, pp. 754-761. https://doi.org/10.1109/TDEI.2013.6518945

\section{General rights}

Copyright and moral rights for the publications made accessible in the public portal are retained by the authors and/or other copyright owners and it is a condition of accessing publications that users recognise and abide by the legal requirements associated with these rights.

Take down policy

If you believe that this document breaches copyright please view our takedown policy at https://edshare.gcu.ac.uk/id/eprint/5179 for details of how to contact us. 


\title{
Application of K-Means Method to Pattern Recognition in On-line Cable Partial Discharge Monitoring
}

\author{
Xiaosheng Peng, Chengke Zhou and Donald M. Hepburn \\ School of Engineering and Built Environment \\ Glasgow Caledonian University \\ Cowcaddens Road, Glasgow, UK, G4 0BA \\ Martin D. Judd and W. H. Siew \\ Department of Electronic \& Electrical Engineering \\ University of Strathclyde \\ Royal College, 204 George Street, Glasgow, UK, G1 1XW
}

\begin{abstract}
On-line Partial Discharge (PD) monitoring is being increasingly adopted in an effort to improve asset management of the vast network of MV and $\mathrm{HV}$ power cables. This paper presents a novel method for autonomous recognition of PD patterns recorded under conditions in which a phase-reference voltage waveform from the $\mathrm{HV}$ conductors is not available, as is often the case in on-line PD based insulation condition monitoring. The paper begins with an analysis of two significant challenges for automatic PD pattern recognition. A methodology is then proposed for applying the KMeans method to the task of recognizing PD patterns without phase reference information. Results are presented to show that the proposed methodology is capable of recognising patterns of PD activity in on-line monitoring applications for both single-phase and three-phase cables and is also effective technique for rejecting interference signals.
\end{abstract}

Index Terms - Autonomous Pattern Recognition, K-Means, Partial Discharge, Phase Resolved Pattern, Cable Condition Monitoring.

\section{INTRODUCTION}

PARTIAL Discharge (PD) is a localized electrical discharge, due to inability of the insulation to withstand the local electrical stress. PD is initiated by a strong electric field and is governed by applied voltage [1]. When the applied voltage is such that it generates an electrical stress greater than local insulation can withstand, breakdown occurs. The localized weakness could be a cavity inside insulation material or surface defect in gaseous insulation, etc (a fuller discussion of defect types can be found in the literature). In the case of internal discharges in a cavity within solid insulation, PD current pulses occur in both the positive and negative half cycles of the applied voltage [2], which is normally a $50 \mathrm{~Hz}$ or $60 \mathrm{~Hz}$ sinusoid in standard AC electrical transmission and distribution systems. The characteristic patterns of PD activity from different types of defect allow them to be distinguished. For decades, Phase Resolved Pattern (PRP) identification (the distribution of PD pulse amplitude with respect to instantaneous phase of the applied voltage) has been one of the key tools for PD based insulation diagnostics [3-5]. With the development of computer technology and mathematical theory, various methods for PD pattern recognition have been developed. At the end of 1960s, CIGRE working group 21.03 published a classic summary of PD recognition [4]. This work addressed factors involved in recognition, diagnosis of PD origin of discharge and identification of external disturbances. Twelve types of typical PD patterns and four additional interference patterns were described in terms of distribution of discharges in each part of the AC cycle and in variation in magnitude with testing voltage and application time. In the 1990s, Krivda introduced a procedure for automated recognition of PD [3], which contained measurements of PD pattern, feature extraction, classification of the pattern and decision process. Cluster analysis and neural networks were applied, based on fundamental feature extraction and database construction. In the $21^{\text {st }}$ century, novel PD pattern recognition methods have continued to develop, including inductive inference algorithm [5], neuro-fuzzy network [6], fractal image compression [7], genetic optimization [8], support vector machine [9] and knowledge-based system [10]. Most of these methods have been applied only within laboratory-based 
experimental settings. No work, as far as the present authors are aware, has reported applying autonomous PD pattern recognition methods in on-line cable $\mathrm{PD}$ monitoring systems. Autonomous PD pattern recognition, having the potential to displace expert-based PD diagnostics through automatic diagnostics, is key to future development of on-line PD monitoring.

In this paper, a novel K-Means based pattern recognition technique is presented. The proposed algorithm aims to provide an automatic pattern recognition method when PD is recorded without voltage phase information. The method is applicable to PDs in single and 3-phase cables, i.e. where electric field at the PD site is influenced by voltages in more than one phase of a 3-phase system. The method is also effective for rejecting interference signals when PDlike interference signals are extracted from noisy raw data.

Validation of the proposed algorithm is carried out using data acquired from cable monitoring systems but it is expected that the algorithm will also be applicable to online PD monitoring of other high voltage apparatus such as transformers, motors, generators and switchgear.

\section{Technical Challenges}

PD pattern recognition, along with steps of data acquisition, data denoising, PD localization and criticality assessment are essential to the success of on-line PD monitoring. In practical on-line situations, two significant challenges must be overcome when attempting autonomous PD pattern recognition in cable condition monitoring. These are addressed in A) and B) below.

\section{A) No voltage phase information}

The first challenge is that a voltage phase reference is difficult to obtain. In power systems, a load-dependent phase shift usually exists between the voltage and current sinusoids. Consequently, the reference phase obtained for current using current transformers or magnetic probes will differ from the voltage phase. For experiments in the laboratory, it is possible to set up a high voltage (HV) coupling capacitor to obtain phase information. However, for on-line applications there are safety and operational constraints which usually prevent connection to a live terminal. Most of the commercial on-line monitoring systems use the phase voltage of the low voltage power supply as a reference, with data acquisition being triggered at the $0^{\circ}$ (positive zero-crossing) point of the low voltage (LV) power supply. Although this LV zero-crossing provides a synchronized reference for data capture, an unknown phase shift will exist relative to the system HV waveform.

Figure 1 shows an example of a set of data acquired from an on-site, in-service measurement of a single core cable feeding a pump motor. The measured raw data is shown in Figure 1 (a); the PD pulses are shown in Figure 1(b), which are extracted by the denoising software developed by the present authors [11, 12]. Given that the PD pulses are expected to be symmetrical in position on the AC cycle waveform, it can be seen that the PD pulses are phase shifted, indicating that data acquisition is not triggered at the $0^{\circ}$ point of the cable voltage. Hence a method of assessing Phase Resolved Patterns from this type of phase-shifted data is required.
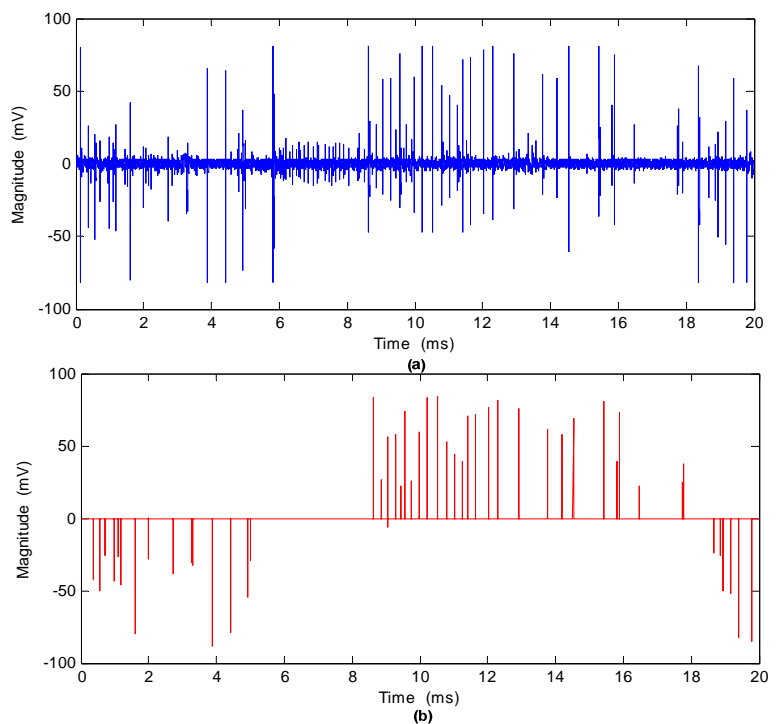

Figure 1 A set of cable PD monitoring data, captured using low voltage supply as trigger. (a) Raw data (b) Extracted PD pulses.

\section{B) PD patterns in three-phase cables}

Most pattern recognition methods have been developed from situations where only a single phase of a system is energised. The second challenge is, therefore, that many on-line systems monitor 3-phase components. In this situation the PD generated can be influenced by the electrical field produced by two or three phase voltages. Analysis of the electric field distribution in a 3-phase cable under a 3-phase symmetrical supply in [11] demonstrates that the fault location determines whether the electrical field stress result from voltages in more than one phase. Figure 2, which shows an example of measured PD pulses from an $11 \mathrm{kV}$ 3-phase Paper Insulated Lead Cable (PILC) cable, illustrates the challenge, which in this case is to automatically extract identifiable patterns where the PD sources may have resulted from the action of 1,2 or all 3 phase voltages.

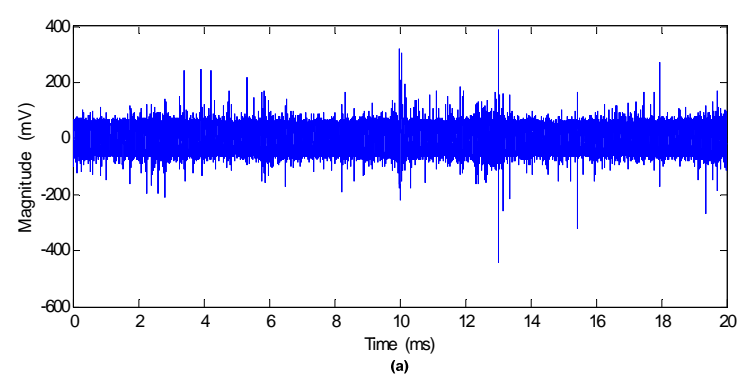




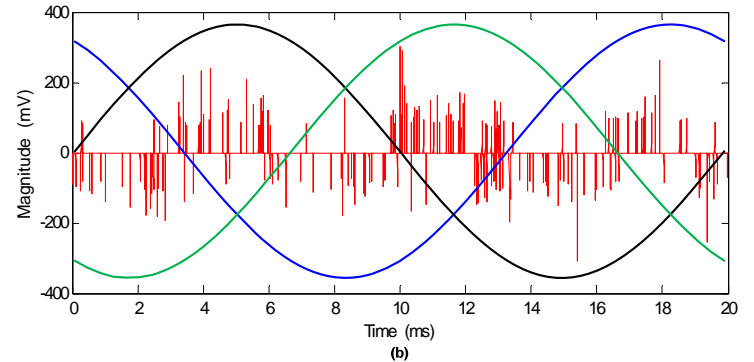

Figure 2. PD activities in an $11 \mathrm{kV}$ three-phase PILC cable captured using low voltage supply as trigger. (a) Raw data (b) Extracted PD pulses.

\section{The K-Means Method}

K-Means is an unsupervised clustering algorithm that is applied in many fields, including pattern classification, multimedia analysis and information retrieval [12-14]. It is a method commonly used to automatically partition a data set into a defined number $(\mathrm{K})$ of groups [15]. The aim of the K-Means algorithm is to separate a number of data points $(M)$ from a set with many dimensions $(N)$ into $K$ clusters so that the within-cluster sum of squares is minimized [16] [17]. The algorithm requires as its input a matrix of $M$ points in $N$ dimensions and a matrix of $K$ initial clusters, also in $N$ dimensions [16]. The flowchart of the KMeans algorithm is divided into 4 steps, which are outlined below.

\section{Step 1: Initialisation of cluster centres.}

Following the method in [16], the points are first ordered by their distances to the overall Mean of the sample. Then, for cluster $L(L=1,2, \ldots, K)$, the $\{1+(L-1) \times[M / K]\}^{\text {th }}$ point is chosen to be its initial cluster centre. In effect, some $\mathrm{K}$ sample points are chosen as the initial cluster centres. This initialization process guarantees that no cluster is empty after initial assignment in the subroutine [16]. Other initialisation methods can be found in [17].

\section{Step 2: Assign each point to nearest 'Means'}

In this step, each point is assigned to the nearest 'Means' which are identified in step 1.

Step 3: Move 'Means' to centre of its cluster

In this step, the centroid of each cluster is calculated: this will be established to be the new 'Means'.

\section{Step 4: Convergence judgement}

The K prototypes $\left(w_{1}, \ldots, w_{k}\right)$ are initialised to one of the $n$ input data $\left(i_{1}, \ldots, i_{n}\right)$. Therefore, $w_{j}=i_{l}, j \in\{1, \ldots, K\}$, $\mathrm{l} \in\{1, \ldots, N\}, C_{j}$ is the $j^{\text {th }}$ cluster whose value is a disjoint subset of input data. The quality of the clustering is determined by the following error function [18]:

$$
E=\sum_{j=1}^{k} \sum_{i_{l} \in C}\left|i_{l}-w_{j}\right|^{2}
$$

Steps 2 and 3 are repeated until $E$ is within defined limits or cluster membership no longer changes, which indicates that the clusters are established and stable. Further details and examples of the method can be found in [19].

\section{K-Means Based PD Pattern Recognition}

Within the thousands of sets of PD data that the authors have examined, during on-site testing and from on-line monitoring data, PD pulses demonstrate cluster characteristic in the time-domain. As K-Means is a simple and effective clustering methodology it has been investigated as a tool for identifying PD patterns in the data.

The basic flowchart of K-Means based PD pattern recognition (which contains a coordinate transform, KMeans based clustering, 'Means' overlaying and PD pattern judgement) is shown in Figure 3. Signal pre-processing, including data denoising [2] [11] [20] is adopted to extract individual PD-like pulses from noisy raw data. The individual pulses are the input of K-Means based PD pattern recognition. It is to be noted that, in addition to PD pulses, this data may contain impulsive noise signals that resemble $\mathrm{PD}$ pulses in the time-domain.

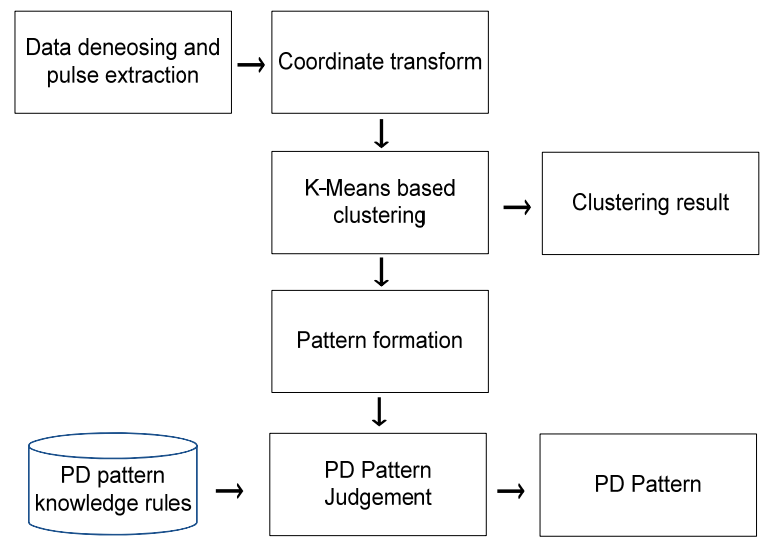

Figure 3. Flowchart for K-Means based PD pattern recognition.

\subsection{COORDINATE TRANSFORM}

The data set shown in Figure 2 is used in this section to illustrate the process of applying the proposed method to PD pattern recognition. As shown in Figure 4, the step of "coordinate transform" turns the time domain representation to one in a polar coordinate where all positive magnitude $\mathrm{PD}$ pulses are slotted in area $\mathrm{A}, \mathrm{B}, \mathrm{C}$ and $\mathrm{D}$, all negative magnitude pulses are drawn in areas $\mathrm{E}$ to $\mathrm{H}$. The reason for projecting positive and negative pulses into separate areas during the mapping is to maintain polarity information during transformation. Coordinate transformation overcomes the first challenge that was discussed in Section 2. This method groups PD in the elliptical system on magnitude and relative phase. If PD pulses exist at the beginning of the collection period then they will be related to those at the end $0^{\circ}$. 

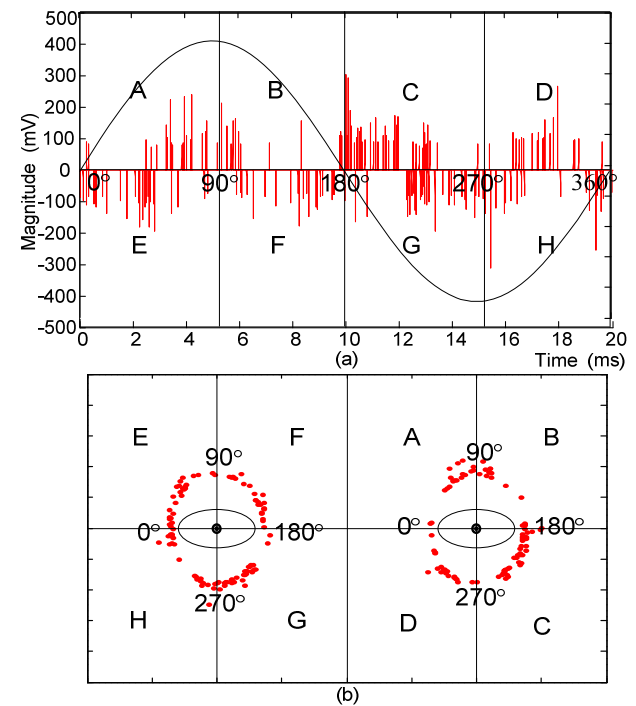

Figure 4. (a) PD pattern in the time-domain, taken from Figure 2; (b) Polar coordinate representation following coordinate transform. Regions A - D represent PD pulses with positive polarity, while $\mathrm{E}-\mathrm{H}$ represent those with negative polarity.

From the observation of on-site test data, when PD exists there are often only a few PD pulses in one set of data. To ensure that statistical relationships are valid, several sets of data are overlaid so that the PD pulses included represent activity over a number of cycles. Effective clustering is difficult to achieve when there are few points in the data set.

\subsection{K-MEANS BASED CLUSTERING}

To first step to address the second challenge discussed in Section 2 of the paper uses the procedure given in Figure 5.

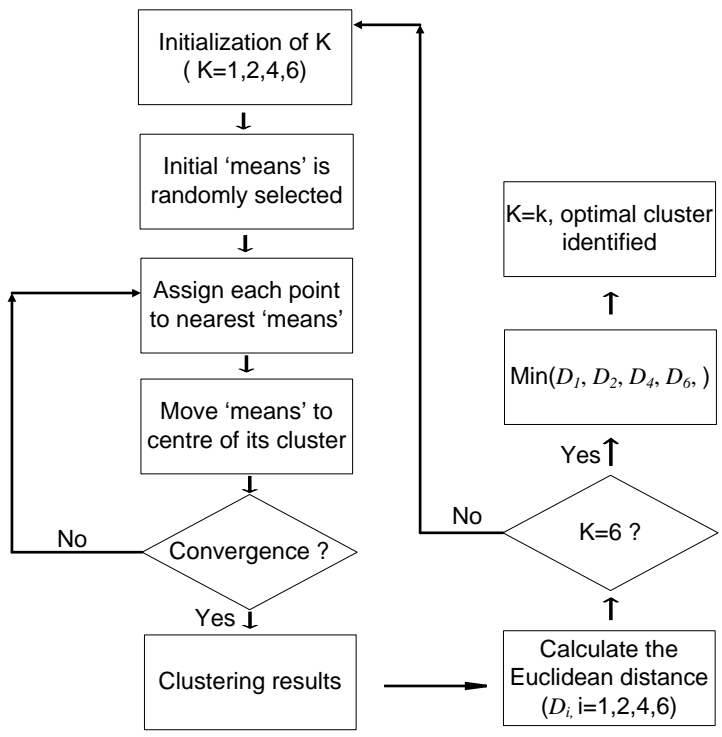

Figure 5. Flowchart of K-Means based clustering.

In Figure 5, the identified optimal cluster $\mathrm{K}$ is related to the type of fault. Therefore, if the optimal cluster is 1 , the data is representative of corona discharge; if the optimal cluster is 2 , data represents internal or surface discharge from one phase voltage; if the optimal cluster is 4 , this indicates internal or surface discharges from two phase voltages and an optimal cluster of 6 indicates internal or surface discharge from all three phases voltage. Then the K-Means method was employed to calculate the number of clusters in the data set selected. The Euclidean distances between the clusters are calculated using (1). After all 4 Euclidean distances $\left(D_{1}, D_{2}, D_{4}\right.$ and $\left.D_{6}\right)$ are available, the minimum distance will be computed, from which the optimal number of clusters $\mathrm{K}$ can be determined.

\subsection{PATTERN FORMATION}

After K-Means based clustering, the next stage of PD pattern recognition (shown in Figure 3 ) is carried out, based on the angles between different 'means'. Before judgement, positive and negative 'means' will be overlaid to enable patterns to be formed. The principle of 'means' overlaying is shown in Figure 6. The original data is shown in Figure 6(a), 'means' in regions $\mathrm{A}, \mathrm{B}, \mathrm{C}$ and $\mathrm{D}$ are combined with those in regions $\mathrm{E}, \mathrm{F}, \mathrm{G}$ and $\mathrm{H}$ respectively. The result of 'means' overlaying is shown in Figure 6(b). In Figure 6(c), the area is divided equally into 6 sub-regions where the angle from $0^{\circ}$ to $60^{\circ}$ is labelled 1 for ease of reference and subsequent $60^{\circ}$ segments are labelled sequentially. Note that the numbering used here is arbitrary.

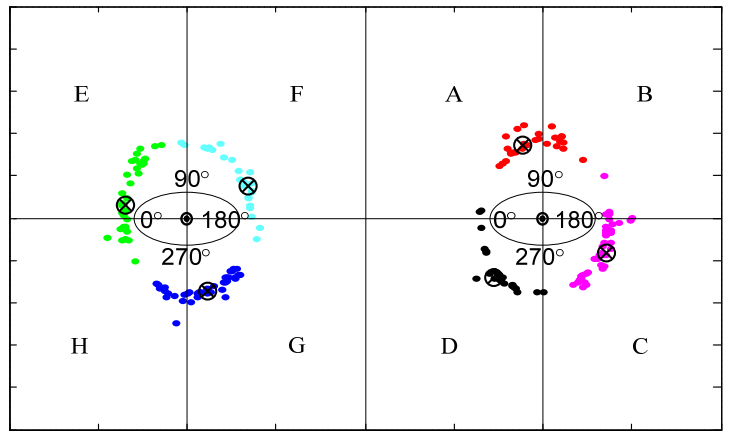

(a)

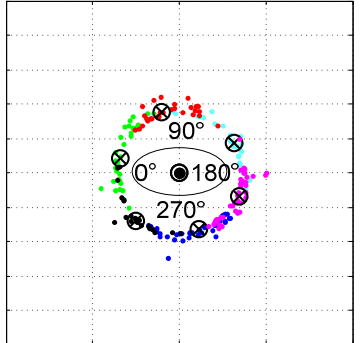

(b)

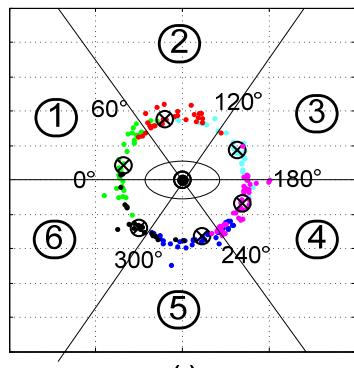

(c)
Figure 6. (a) Before 'means' overlaying (b) After 'means' overlaying (c) The area is divided into 6 sub-regions labelled (1) - (6).

\subsection{AUTONOMOUS PD PATTERN JUDGEMENT}

After the process above is complete, the following five criteria are used for PD pattern recognition. Figures 7-10 illustrate the time-domain distribution of PD pulses and 
their respective diagnoses following processing using the proposed method.

Criterion 1: If $\mathrm{K}=1$, is the data represents a corona discharge.

Criterion 2: If $\mathrm{K}=2$ and angles of 'means' between areas is $180^{\circ} \pm 10 \%$, e.g. areas 1 and 4 , an internal or surface discharge due to the effect of one phase voltage is identified.

Criterion 3: If $\mathrm{K}=4$ and angle between neighbouring 'means' is either $60^{\circ} \pm 10 \%$ or $120^{\circ} \pm 10 \%$, internal or surface discharge due to the effect of two phase voltages is identified.

Criterion 4: If $\mathrm{K}=6$ and angle between neighbouring 'means' is $60^{\circ} \pm 10 \%$, internal discharge or surface discharge due to the effect of all three phase voltages is identified.

Criterion 5: No PD pattern exists if criteria 1-4 are not satisfied.

It can be argued that when $\mathrm{K}$ is 2 , it can indicate corona from 2 phases and when $\mathrm{K}$ is 3 it may be due to corona from 3 phases. However the degree of separation will help to distinguish between PDs and corona.

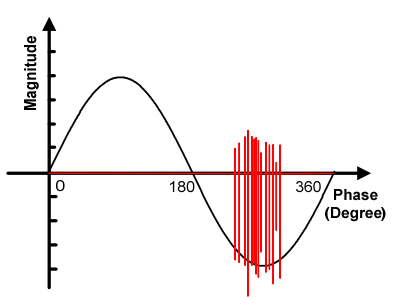

(a)

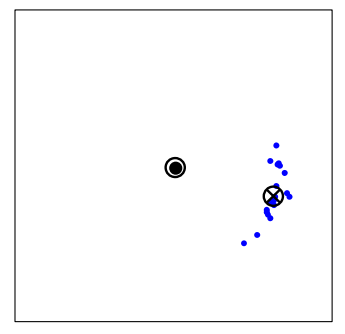

(b)
Figure 7. (a) A typical corona discharge in time domain, and (b) its KMeans clustering result.

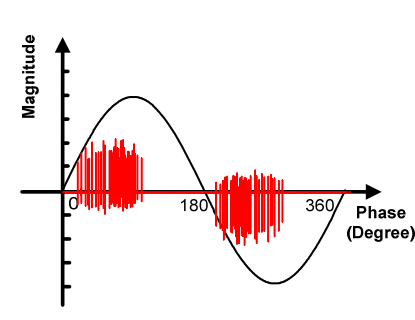

(a)

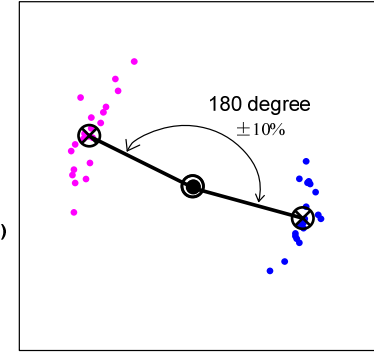

(b)
Figure 8. (a) A typical internal discharge or surface discharge affected by one phase voltage, and (b) its K-Means clustering result.

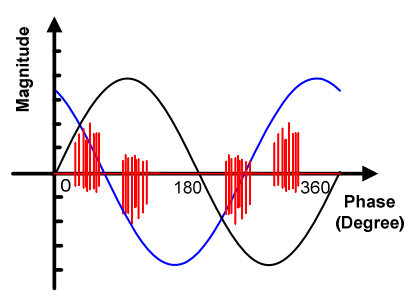

(a)

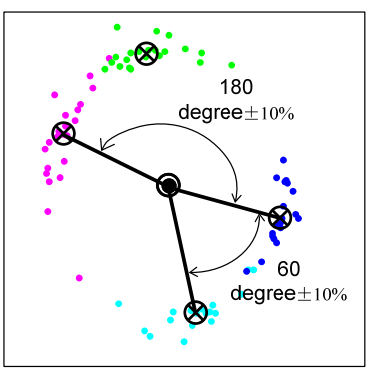

(b)
Figure 9. (a) A typical internal discharge or surface discharge affected by two phases voltage (b) K-Means clustering result.

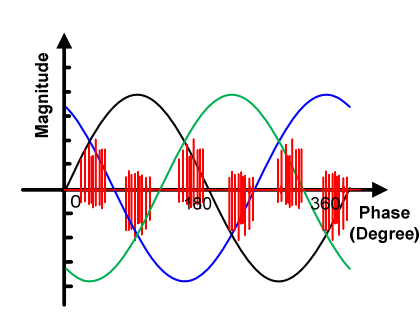

(a)

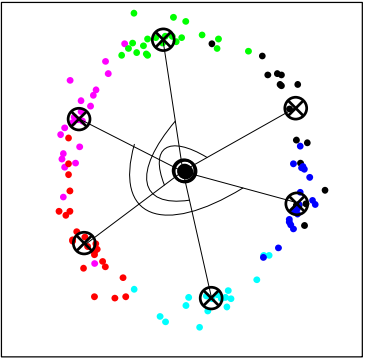

(b)
Figure 10. (a) A typical internal discharge or surface discharge affected by three phases voltage (b) K-Means clustering result.

\section{Application Examples}

Five examples, taken from on-site PD measurements in substations using portable PD capturing systems or from on-line PD monitoring systems, are presented to evaluate the proposed autonomous PD pattern recognition algorithm. Signals from 4 examples are successfully identified by the method to be PD patterns, i.e. corona discharge, PD resulting from one phase voltage, PD from 2 phase voltages and PD from 3 phase voltages. One example of regular interference signals is successfully identified as non-PD, which demonstrates another significant functionality of the method, further interference signals rejection.

\subsection{CORONA DISCHARGE $(K=1)$}

The first example was acquired from an on-site testing of Cross-Linked Polyethylene (XLPE) cable system. For the signals shown in Figure 11(a) are raw data; signals shown in Figure 11 (b) are the extracted PD pulses. Figure 11(c) shows the result of 4 sets of data combined. Corona discharge has been recognised as the number of means $\mathrm{K}$ is determined as 1 .

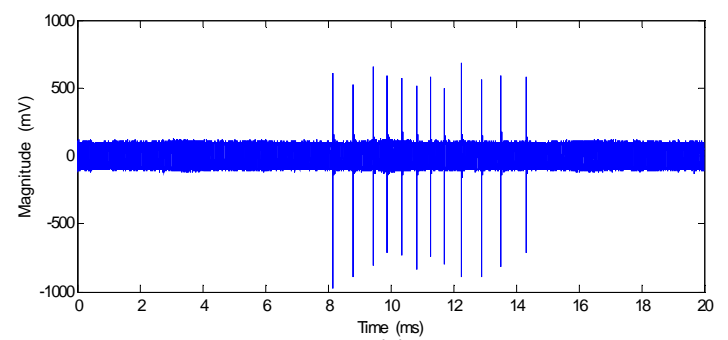

(a) 


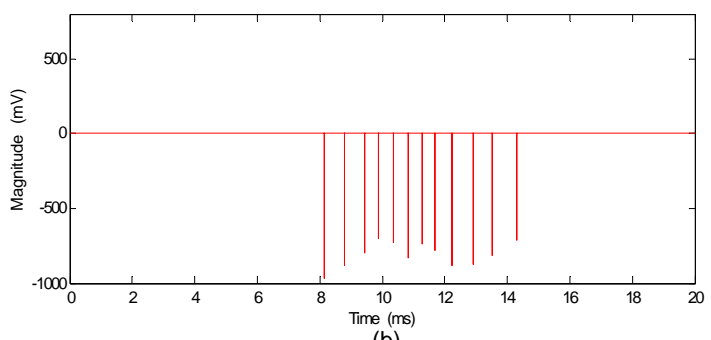

(b)

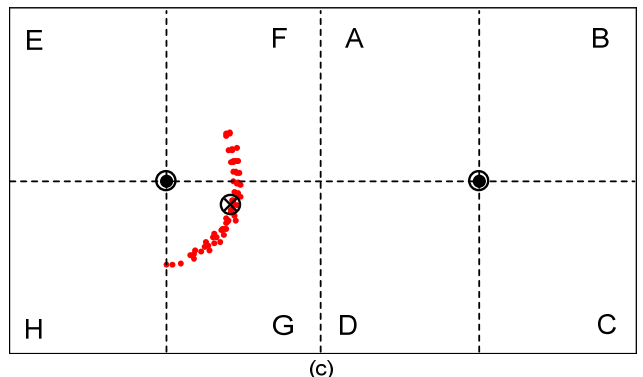

Figure 11. (a) Raw data (b) Extracted PD pulses (c) K-Means based clustering recognition of 4 sets of data.

\subsection{PD FROM A MOTOR (K=2)}

An example of PD from a motor, which was acquired from an on-site testing of an Ethylene Propylene Rubber (EPR) cable circuit, to which the motor is connected at the far end, is shown in Figure 12. The raw data and extracted PD pulses are those shown earlier in Figure 1. There is a phase shift between the low voltage with which the zerocrossing was used to trigger the data acquisition system, and the $11 \mathrm{kV}$ supply in the cable. Figure 12 shows that the number of means, $\mathrm{K}$, has been determined as 2 . Using the criteria presented in the last section of the paper, the PD activity is due to either an internal discharge or a surface discharge emanating from a single phase.

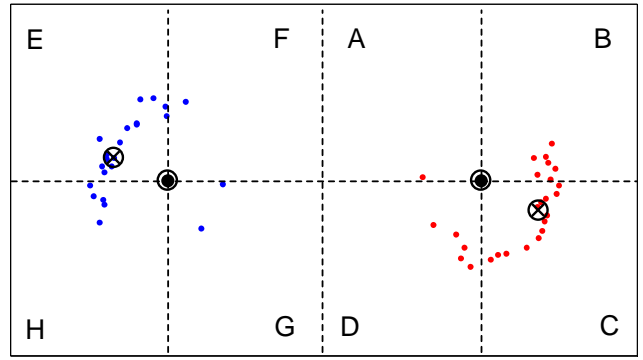

Figure 12. K-Means based clustering recognition in processing of a set of cable PD monitoring data in a power station.

\subsection{INTERNAL DISCHARGE (K=4)}

Another example of internal discharge, which was acquired from a PILC cable on-line monitoring system, is shown in Figure 13. Raw data is shown in Figure 13(a). Extracted PD pulses are shown in Figure 13(b). Figure 13(c) shows the results of one set of data, 4 clusters are calculated. Figure 13(d) shows the result of 4 sets of overlapped data and the number of means, $\mathrm{K}$, has been determined as 4 .

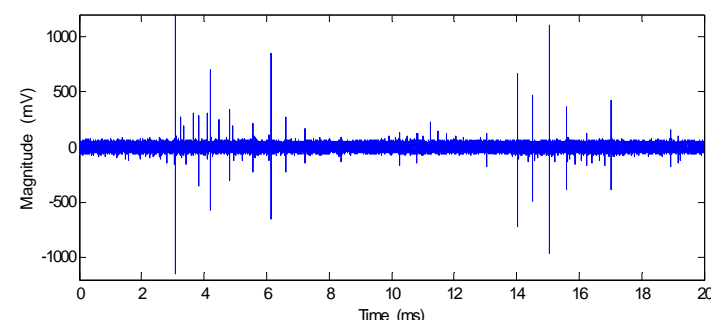

(a)

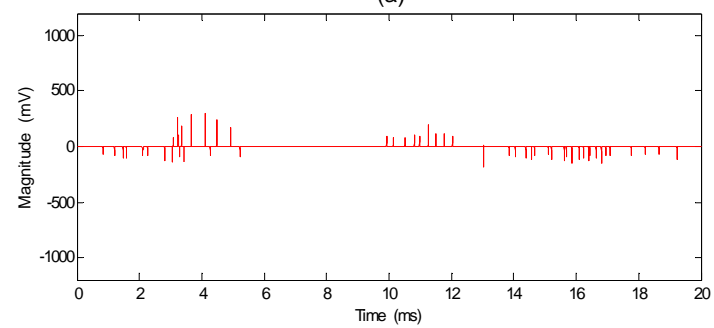

(b)
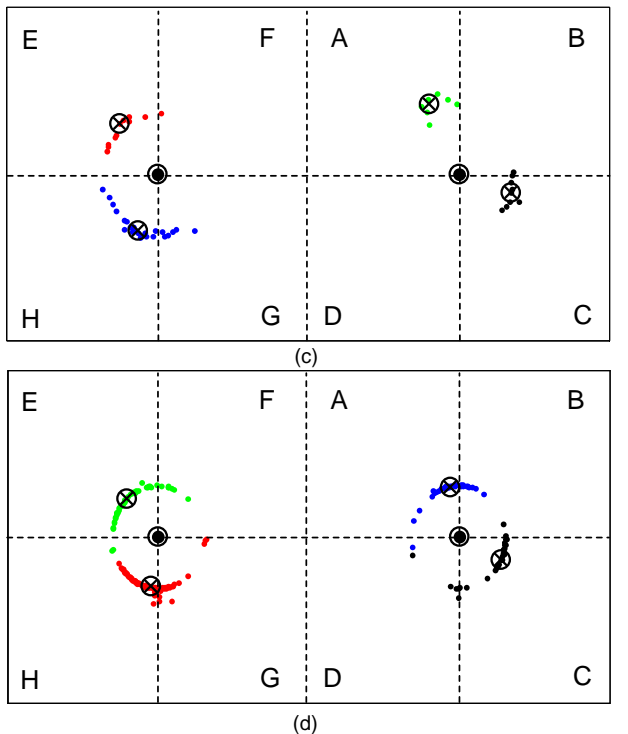

Figure 13. (a) Raw data (b) Extracted PD pulses (c) K-Means based clustering recognition of data set of cable monitoring data (d) K-Means based clustering recognition of 4 sets of data.

\subsection{INTERNAL DISCHARGE $(\mathrm{K}=6)$}

An example of an internal discharge, which was acquired from a PILC cable on-line monitoring system, is shown in Figure 14. Raw data is shown in Figure 14(a). Extracted PD pulses are shown in Figure 14(b). Figure 14(c) shows the results of one set of data, no cluster is calculated. Figure 14(d) shows the result of 4 sets of overlapped data, for which 6 clusters are identified.

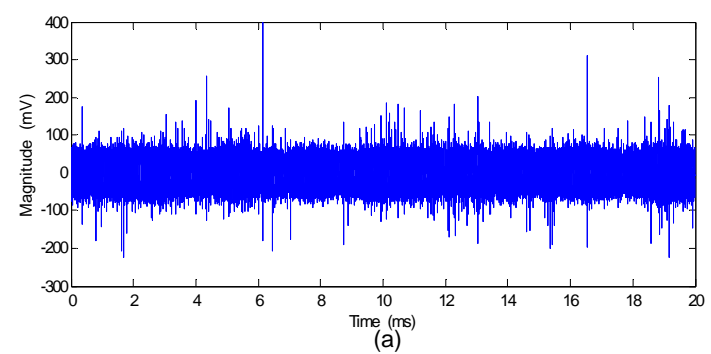




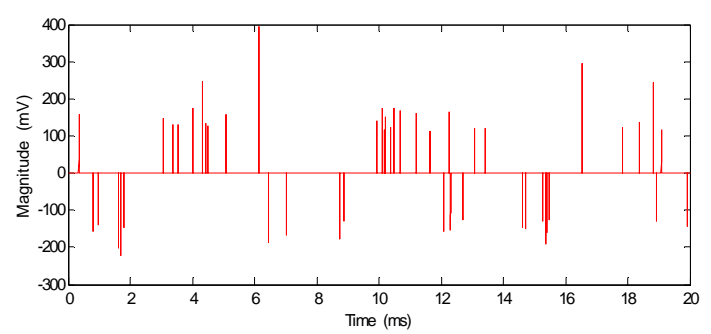

(b)
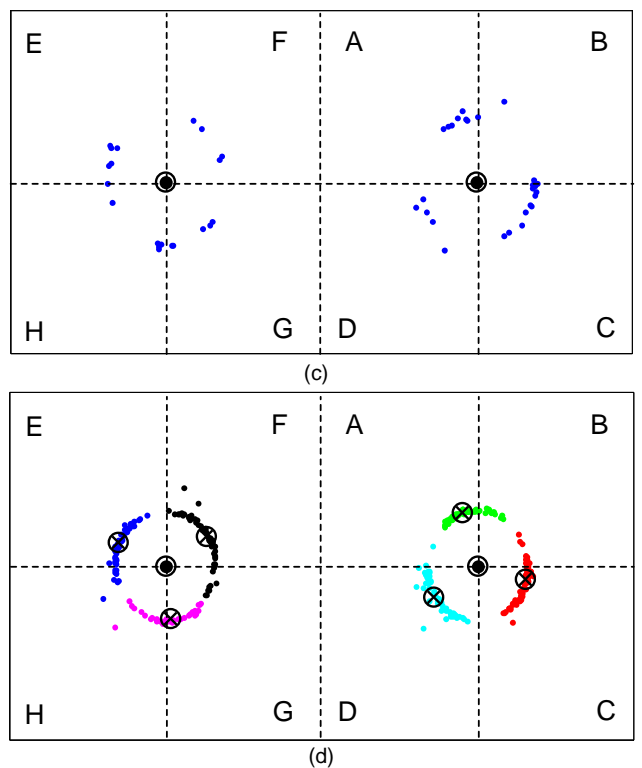

Figure 14. (a) Raw data (b) Extracted PD pulses (c) K-Means based clustering recognition of data set of cable monitoring data (d) K-Means based clustering recognition of 4 sets of data.

\subsection{REGULAR INTERFERENCE SIGNAL}

An example of a regular interference signal, recorded during on-site testing of the EPR cable system, is shown in Figure 15. Raw data is shown in Figure 15(a). Extracted PD pulses are shown in Figure 15(b). Figure 15(c) shows the results of one set of data, no cluster has been recognised, which indicates that the signals are interference, not PD.
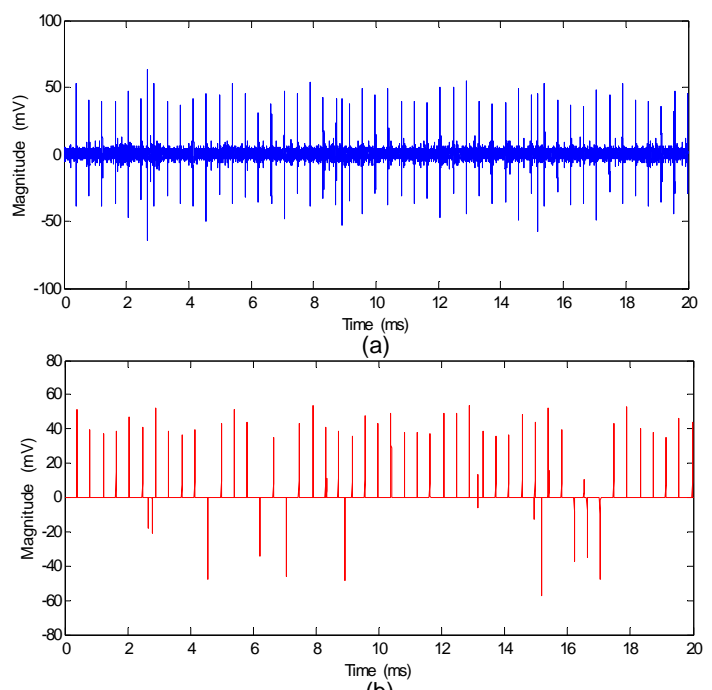

(b)

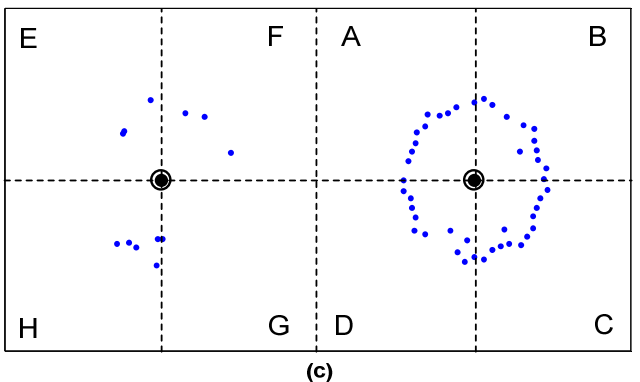

Figure 15. (a) Raw data (b) Extracted PD pulses (c) K-Means based clustering recognition.

The proposed K-Means based PD pattern recognition algorithm has been tested against 85 sets of on-line monitoring data. Without signal overlaying, 68 sets of data were recognized successfully, giving a recognition accuracy rate of $80 \%$. However, when signals were overlayed from a minimum of 5 AC cycles of monitoring data, the success rate improved to $100 \%$.

\section{Conclusions}

A novel, K-Means based, autonomous phase resolved PD pattern recognition method has been presented and demonstrated to have the following advantages:

- The method can automatically recognise PD patterns when phase information is not available.

- A recognition rate of $80 \%$ was achieved when the algorithm was tested on 85 sets of on-line monitoring data, when signal overlaying is not employed. However, accuracy increased to $100 \%$ when overlaying of 5 cycles of data records, demonstrating that the method is highly effective.

- The method is applicable to three phase cable systems when PD patterns can be the result of voltages from a single phase, two of the three phases or all three phases. It is capable of recognising various PD patterns.

- The method is effective for further rejection of PD-like pulse-shaped interference signals. However, effective denoising is required, prior to application of the method, to remove continuous radio signal interference and white noise.

Also, the method is not without limitations, and further work is required to develop a capability to distinguish between internal discharge and surface discharge and to recognise patterns associated with multiple sources of PD.

\section{ACKNOWLEDGEMENT}

The authors would like to thank EPSRC (UK) for funding the research, under grants EP/G028397/1 and EP/G029210/1. 


\section{REFERENCES}

[1] IEC International Standard 60270. "High Voltage Test Techniques Partial Discharge Measurements”, International Electrotechnical Commission (IEC), Geneva, Switzerland, 3rd edition, 2000.

[2] X. Zhou, “An Optimal Wavelet-Based Algorithm for Noise Rejection in PD Measurement”, PhD Thesis, Glasgow Caledonian University, UK, 2006.

[3] A. Krivda, "Automated Recognition of Partial Discharges", IEEE Trans. Dielectrics and Electrical Insulation, Vol. 2, No. 5, pp. 796821, October 1995.

[4] Working Group 21.03 Cigre, "Recognition of Discharges”, Electra, Vol. 11, pp. 61-98, 1969.

[5] T. K. Abdel-Galil, R. M. Sharkawy, M. M. A. Salama, R. Bartnikas, "Partial Discharge Pulse Pattern Recognition using an Inductive Inference Algorithm”, IEEE Trans. Dielectrics and Electrical Insulation, Vol. 12, No. 2, pp. 320-327, April 2005

[6] C. Mazzetti, F. Mascioli, F. Baldini, M. Panella, R. Risica, R. Bartnikas, "Partial Discharge Pattern Recognition by Neuro-Fuzzy Networks in Heat-Shrinkable Joints and Terminations of XLPE Insulated Distribution Cables”, Trans. Power Delivery, Vol. 21, No. 3, pp.1035-1044, July 2006.

[7] J. Li, C. Sun, "Partial Discharge Image Recognition Influenced by Fractal Image Compression”, IEEE Trans. Dielectrics and Electrical Insulation, Vol. 15, No. 2, pp.496-504, April 2008

[8] A. Rizzi, F. Massimo, F. Mascioli, F. Baldini, C. Mazzetti, R. Bartnikas, "Genetic Optimization of a PD Diagnostic System for Cable Accessories”, Transactions on Power Delivery, Vol. 24, No. 3, pp. 1728-1738, July 2009.

[9] L. Hao, P. L. Lewin, "Partial Discharge Source Discrimination using a Support Vector Machine”, IEEE Trans. Dielectrics and Electrical Insulation, Vol. 17, No. 1, pp. 189-197, February 2010.

[10] S. Rudd, S. D. J. McArthur, M. D. Judd, S. Meijer, E. Gulski, "Knowledge-based diagnosis of partial discharges in power transformers”, IEEE Trans. Dielectrics and Electrical Insulation, Vol. 15, No. 1, pp. 259-268, February 2008.

[11] C. Zhou, M. Michel, D. M. Hepburn, X. Song, "On-line partial discharge monitoring in medium voltage underground cables”, IET Science, Measurement and Technology, Vol.3, Iss.5, pp. 354-363, September 2009.

[12] T.W. Chen and S.Y. Chien, "Bandwidth Adaptive Hardware Architecture of K-Means Clustering for Video Analysis”, IEEE Transactions on Very Large Scale Integration (VLSI) Systems, Vol. 18, No.6, pp. 957-966, June 2010.

[13] P. Bellot, M. El-Beze, "A clustering method for information retrieval”, (Technical Report IR-0199), Laboratoire d'Informatique d'Avignon, France, 1999.

[14] D. M. Mount, N. S. Netanyahu, C. D. Piatko, R. Silverman, A.Y. Wu. "An Efficient K-Means Clustering Algorithm: Analysis and Implementation”. IEEE Transactions on Pattern Analysis and Machine Intelligence, Vol. 24, No. 7, pp. 881-892, July 2002.

[15] J. B. MacQueen, "Some methods for classification and analysis of multivariate observations", Proceedings of the Fifth Symposium on Math, Statistics, and Probability. Berkeley, CA: University of California Press, pp. 281 - 297, 1976.

[16] J. A. Hartigan, M. A. Wong, “A K-Means Clustering Algorithm”, Journal of the Royal Statistical Society. Series C (Applied Statistics), Vol. 28, No. 1(1979), pp. 100-108, 1979.

[17] P. S. Bradley, U. M. Fayyad, "Refining Initial Points for K-Means Clustering”, 15th International Conference on Machine Learning (ICML98), Morgan Kaufmann, San Francisco, 1998.

[18] N. Shi, X. M. Liu, Y. Guan, "Research on k-means Clustering Algorithm: An Improved k-means Clustering Algorithm”, Third International Symposium on Intelligent Information Technology and Security Informatics (IITSI), pp.63-67, April 2010.

[19] M. K. Ng, Y. -m. Cheung, J. Z. Huang, “Agglomerative Fuzzy KMeans Clustering Algorithm with Selection of Number of Clusters”, IEEE Transactions on Knowledge and Data Engineering, Vol. 20, No. 11, pp. 1519-1534, November 2008.

[20] X. Song, C. Zhou, D.M. Hepburn and M. Michel, "Second Generation Wavelet Transform in PD Measurement Denoising”, IEEE Trans. Dielectrics and Electrical Insulation, Vol. 14 No. 6, December 2007.

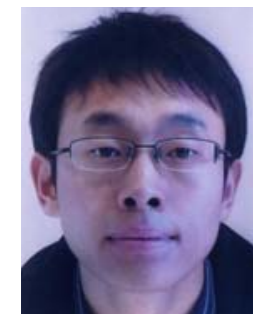

Xiaosheng Peng received the B.Sc. M.Sc. degrees in hydropower and information engineering from Huazhong University of Science and Technology, China in 2006 and 2009 respectively, and Ph.D. in electrical engineering at Glasgow Caledonian University in 2012. He is currently a research assistant in Glasgow Caledonian University. His research interests are partial discharge signal processing and condition monitoring of power plant. He is a member of IEEE.

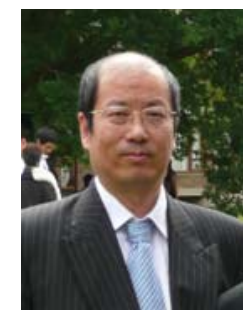

Chengke Zhou received the B.Sc. and M.Sc. degrees in electrical engineering from HuaZhong University of Science and Technology, China in 1983 and 1986, respectively, and the Ph.D. degree at the University of Manchester U.K., in 1994. Since then, he worked in Glasgow Caledonian University, U.K., as a Lecturer, Senior Lecturer and in Heriot-Watt University as a Reader until 2007 when he went back to Glasgow Caledonian University as a Professor. He has published more than 100 papers in the area of partial discharge based condition monitoring of $\mathrm{MV} / \mathrm{HV}$ plant and power system analysis. He is member of IEEE(06) and IET.

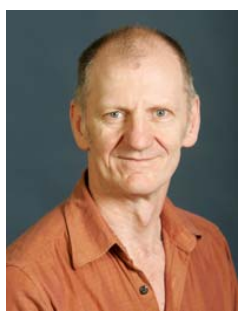

Donald M. Hepburn received his B.A. (Hons) from the Open University in 1987and the Ph.D. degree from Glasgow Caledonian University (GCU) in 1994.He has many years of industrial research experience and has been involved in research into high voltage insulation systems at GCU for over 20 years. His research interests cover monitoring of chemical changes to insulation materials, application of electrical, acoustic and RF monitoring equipment to $\mathrm{HV}$ components and application of advanced digital signal processing to information from the monitoring techniques. He is a Senior Lecturer at GCU and is involved in industrial and academic research projects.

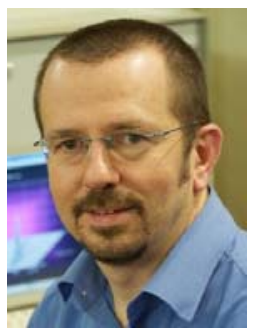

Martin D. Judd (M'02, SM'04) graduated from the University of Hull in 1985 with a degree in Electronic Engineering. He subsequently gained 8 years of industrial experience within the GEC group of companies. Martin received his $\mathrm{PhD}$ from the University of Strathclyde in 1996 for research into partial discharges in gas insulated switchgear. His fields of interest include high frequency electromagnetics, generation and measurement of fast transients, partial discharges and energy harvesting. Dr Judd is a Reader in the Institute for Energy and Environment at the University of Strathclyde, a Chartered Engineer, a Member of the IET and Senior Member of the IEEE.

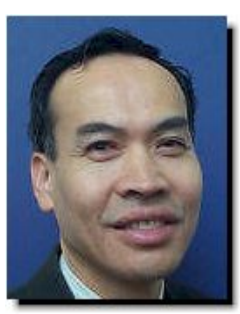

W. H. Siew is a Reader in the Department of Electronic \& Electrical Engineering, University of Strathclyde, Glasgow, Scotland. He is a triple alumnus of the University of Strathclyde with degrees in B.Sc. (Hons) in Electronic \& Electrical Engineering; $\mathrm{PhD}$ in Electronic \& Electrical Engineering; and Master of Business Administration. His areas of research interest include large systems electromagnetic compatibility; cable diagnostics; lightning protection; and wireless sensing systems. He is Convener of the CIGRE WG C4.208 and a member of the Technical Advisory Panel for the IET Professional. He is also a member of IEEE TC7. He is a Chartered Engineer and an MIEE and an MIEEE. 